\title{
Relationship and Priority of Risks using ISM and Grey Approach
}

\author{
KyoungJong Park \\ Dept. of Business Administration, Gwangju University \\ 277 Hyoduk-Ro, Nam-Gu, Gwangju 61743, KOREA \\ kjpark@gwangju.ac.kr
}

\begin{abstract}
A supply chain should ensure survivability and sustainability. However, a supply chain has been exposed to various risks because of uncertainty, complexity, ambiguity, and etc. These risks expose the vulnerability of a supply chain and threaten the survivability and sustainability of the chain. If a risk occurs in a supply chain, it should be removed or weaken from the supply chain. But, it is not easy to remove or weaken from the chain. Therefore, this study proposes a method to prioritize the risks and to establish a corresponding strategy about them after considering the relationship and fatality of the risks which occur in a supply chain. This study uses Grey approach and Interpretive Structural Modeling (ISM) to propose a method which finds the location, the most vulnerable place, about the risks in a supply chain.
\end{abstract}

Keywords: Survivability, Sustainability, Uncertainty, Complexity, Vulnerability, Risk, Grey approach, Interpretive structural modeling

\section{Introduction}

A supply chain should ensure survivability and sustainability. However, a supply chain has been exposed to various risks because of uncertainty, complexity, ambiguity, and etc. According to the increasing of social complexity and uncertainty, it is great on not only the sustainability of a company, but also the sustainability of the supply chain that includes the company $[1,2,3,4,5]$. The sustainability of a supply chain is differently influenced by the counter strategy of companies in a supply chain about risks.

The concept of risk in a supply chain is still vague and often ill-defined and Heckmann et al., [6] expressed risk as "the fear that economic activities lead to the loss or devaluation of an important asset or a decrease in the performance of the business". March \& Shapira [7] defined supply chain risk as "variation in the distribution of possible supply chain outcomes, their likelihood, and their subjective values". Peck [8] defined supply chain risk as "anything that (disrupts or impedes) the information, material or product flows from original suppliers to the delivery of the final product to the ultimate end user". Venkatesh et al., [9] classified that "risk is measurable and can be estimated from the probabilities of the outcome" and Knignt [10] classified that "uncertainty is not quantifiable and probabilities of the outcome are not known". But Yates \& Stone [11] argued that "risk implies uncertainty about the prospective outcome and if the probability of the outcome is known then there is no risk".

Christopher et al., [12] defined supply chain risk as "any risk to material, product and information flow from the original supplier to the delivery of the final product". Supply chain risk is defined as three general types of risk or threats which impact the supply chain: natural accidents (fire, earthquake, etc.), normal accidents (technology failure/breakdowns), and abnormal accidents (ill-will by insiders/outsiders) [13].

Received (June 18, 2017), Review Result (August 24, 2017), Accepted (August 30, 2017) 
A supply chain is easily exposed to various risks and the type and intensity of risks are different by the circumstance of a supply chain. The risk which occurs in a supply chain should managed by the concept of supply chain risk management (SCRM). SCRM is defined as "the implementation of strategies to manage both every day and exceptional risks along the supply chain based on continuous risk assessment with the objective of reducing vulnerability and ensuring continuity" [14].

Basically, a supply chain uses the preventive approach before a risk occurs and it uses the interceptive approach after a risk is realized. But, a supply chain cannot prevent all risks which occur in advance and cannot recover the status of a supply chain into the status before there is any risk. Therefore, it may be realistic to find the most vulnerable position in a risk and then manage it after considering the possibility and impact of a risk. This study will propose a priority rule about a risk in a supply chain in order to select the most vulnerable position of the chain considering the impact of the risk which is possible to occur. This paper applies Interpretive Structural Modeling (ISM) and Grey approach to find the most vulnerable position about a risk which occurs in a supply chain.

This paper explained that a risk in a supply chain and the research purpose in Section 1 and will explain the related researches to select the priority of a risk in Section 2. In Section 3, this study will propose a method to select the most vulnerable position about a risk in a supply chain and in Section 4 , the proposed method will be examined by a numerical example and the result is analyzed. Finally, the conclusion and future researches will be proposed in Section 5.

\section{Related Researches}

Supply chain risks are diverse and the risks depend on the environment and condition of a company. And the effect of a risk to the company is different. Business Continuity Institute Survey [15] explained the top three causes of supply chain disruptions as unplanned IT \& telecommunications outage (64\%), cyberattack \& data breach (54\%), adverse weather $(50 \%)$ and etc., (Figure 1).

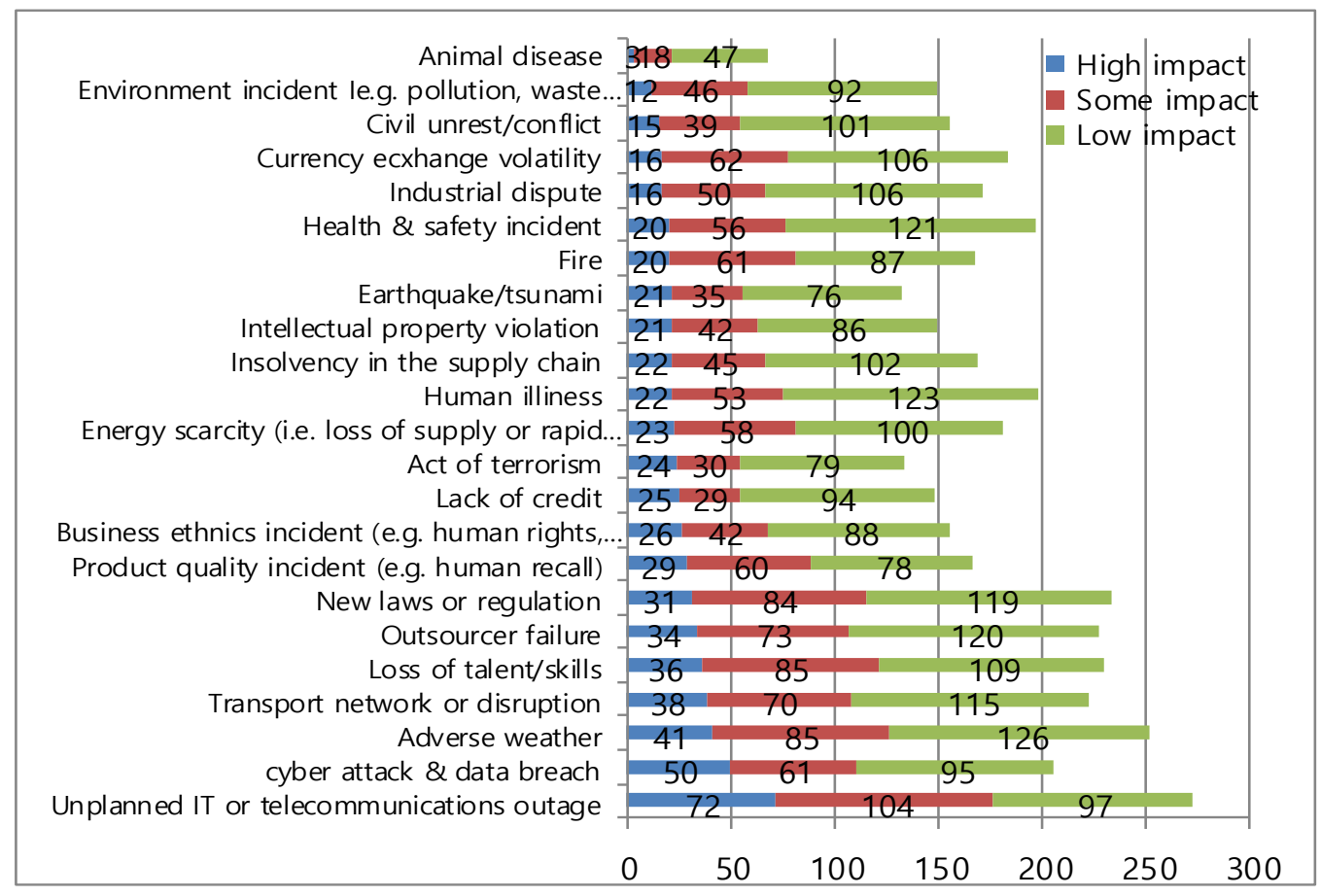

Figure 1. Causes of Supply Chain Disruption 
Also, Business Continuity Institute Survey [15] explained the consequences of supply chain disruptions as loss of productivity (58\%), customer complaints received (40\%), increased cost of working (39\%), loss of revenue (38\%), service outcome impaired (36\%), and etc. (Figure 2). Business Continuity Institute Survey [15] revealed that "the most common consequences of disruption such as the loss of productivity (58\%), customer complaints $(40 \%)$, and increased cost of working (39\%). The percentage of responses indicating the consequences of supply chain disruption falls across the board this year, notably damage to brand reputation (34.6\% to $27 \%$ ) and delayed cash flows $(34.1 \%$ to $21 \%)$. The only exception is product recall or withdrawal $(6.5 \%$ to $10 \%)$."

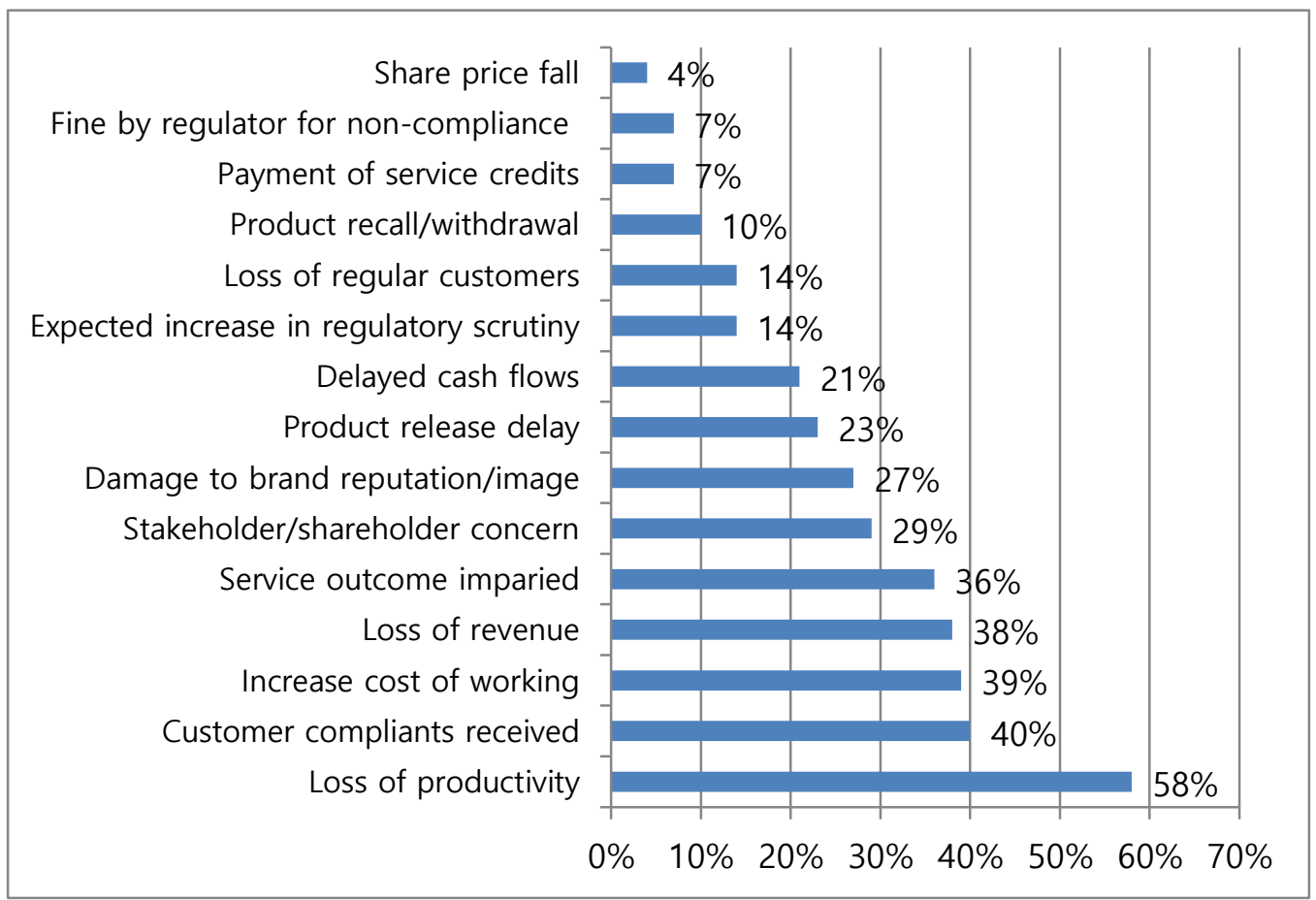

Figure 2. Consequences of Supply Chain Disruption

As shown in Figures 1 and 2, the causes of a risk in a supply chain are various and the results of a risk are various. So, in the view of a supply chain, it is difficult to detect and remove all of the cause of a risk. Also, it is necessary to calculate the effect of a risk quantitatively to remove a risk in a supply chain. The study proposed quantitative risk measures as standard deviation, mean-variance approaches, value-at-risk (VaR), conditional-value-at-risk ( $\mathrm{CVaR})$, premiums, likelihood, the severity of adverse effects, and the extent of loss to measure a supply chain [6]. Khan et al. [16] explained the models for the prioritization of risk as Failure Mode Effect Analysis (FMEA), Risk Benefit Analysis (RBA), and Cost Benefit Analysis (CBA). The models are based on factors like the probability of occurrence, severity, and the detection ease.

Because risks that occur in a supply chain take affect each other, a risk cannot simply be considered as the problem of only the risk. It is possible to accurately measure the impact of a risk after the impact it receives from another risk and gives to another risk should be considered at the same time. ISM is one of the ways that measure objectively the impact among risks.

ISM is defined as a process which modifies the unclear and poorly articulated mental models of systems into the visible, well-defined models useful for many purposes $[9,17]$. That is, if ISM is used in the evaluations of risks in a supply chain, it takes into account 
the interdependency between the various risks with the meaning of showing a driver risk of multiple other risks $[9,18,19]$.

Grey system theory has been studied to overcome the vague and ambiguous phenomenon and to propose an objective solution [20, 21]. Memon et al. [22] applied Grey system theory and uncertainty theory for selecting suppliers in a supply chain and Baskaran et al. [23] applied Grey approach to evaluate the sustainability of textile providers. Brito et al. [24] applied Grey-based ELECTRE approach to sort risk factors in a supply chain.

\section{The Prosed Method}

This paper applies 2 Stages as follows in Figure 3 to increase the accuracy for selecting the risk priority in a supply chain. In Stage 1, this study identifies the association between 2 risks using ISM. In Stage 2, this article determines linguistic variables and Grey numbers for each attribute after giving the relevant information between 2 risks to experts and applies Grey approach using the Grey numbers. Finally, it selects the location which influences on the most serious effect.

ISM algorithm used in Stage 1 can be described as follows in Table 1 to determine the relationship among risks $[9,25]$. ISM is used instead of the traditional cause effect, fishbone, Ishikawa diagram, and etc. because it is easier to understand the relationship among various interrelated factors which lead a particular effect [9].

Stage 1:

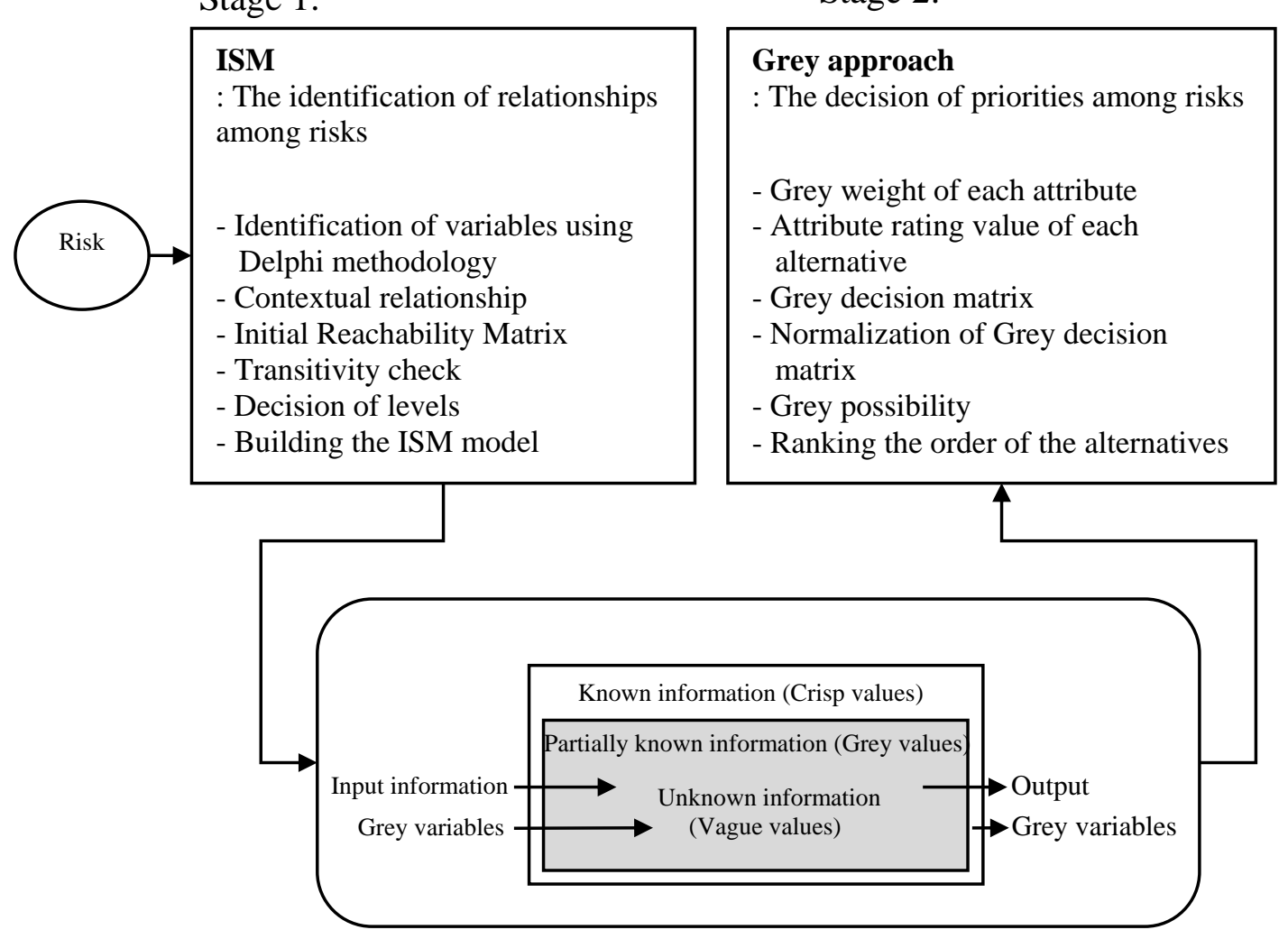

\section{Stage 2:}

- Grey weight of each attribute

Attribute rating value of each matrix

Grey possibility

- Ranking the order of the alternatives
: The decision of priorities among risks

Figure 3. A Proposed Algorithm using ISM and Grey Approach 
Table 1. Stage 1. The Algorithm of ISM

\begin{tabular}{|c|c|}
\hline Step & Explanation \\
\hline $\begin{array}{l}\text { 1: Identification of } \\
\text { variables }\end{array}$ & $\begin{array}{l}\text { Key parameters of the system are determined by checking through a } \\
\text { literature survey and brain storming (in this paper, Focus Group } \\
\text { Interview (FGI)) with the experts. }\end{array}$ \\
\hline $\begin{array}{l}\text { 2: Constitution of } \\
\text { contextual } \\
\text { relationship }\end{array}$ & $\begin{array}{l}\text { Contextual relationship identifies the pairs of variables for variables } \\
\text { which are determined in Step } 1 \text {. Contextual relationship is a matrix } \\
\text { which is called as a structural self-interaction matrix (SSIM). } \\
\text { The notations used to develop SSIM are as follows. } \\
\text { F: Risk variable } \mathrm{i} \text { leads to variable } \mathrm{j} \\
\text { B: Risk variable } \mathrm{j} \text { leads to variable } \mathrm{i} \\
\text { T: Risk variable } \mathrm{i} \text { leads to variable } \mathrm{j} \text { and vice versa } \\
\mathrm{N} \text { : No relationship between the variables }\end{array}$ \\
\hline $\begin{array}{l}\text { 3: Constitution of } \\
\text { Initial Reachability } \\
\text { Matrix }\end{array}$ & $\begin{array}{l}\text { In SSIM, F, B, T, and N are replaced by } 1 \text { or } 0 \text { by the following rule. } \\
\text { SSIM is converted to a binary matrix called the initial reachability } \\
\text { matrix. } \\
\text { Rule } 1 \text { : If the }(i, j) \text { entry in the SSIM is } F \text {, then the }(i, j) \text { entry in the } \\
\text { reachability matrix becomes } 1 \text { and the }(j, i) \text { entry is } 0 \text {. } \\
\text { Rule } 2 \text { : If the }(i, j) \text { entry in the SSIM is } B \text {, then the }(i, j) \text { entry in the } \\
\text { reachability matrix is } 0 \text { and the }(j, i) \text { entry becomes } 1 \text {. } \\
\text { Rule } 3 \text { : If the }(i, j) \text { entry in the SSIM is T, then the }(i, j) \text { entry in the } \\
\text { reachability matrix becomes } 1 \text { and the }(j, i) \text { entry also becomes } 1 \text {. } \\
\text { Rule } 4 \text { : If the }(i, j) \text { entry in the SSIM is } N \text {, then the }(i, j) \text { entry in the } \\
\text { reachability matrix becomes } 0 \text { and the }(j, i) \text { entry also becomes } 0 \text {. }\end{array}$ \\
\hline 4: Transitivity check & $\begin{array}{l}\text { Transitivity check inspects the reachability matrix created by SSIM } \\
\text { and constitutes the transitivity matrix. } \\
\text { Transitivity check means that if a variable, a, is related to a variable, } \\
\text { b, and if the variable, b, is related to a variable, c, and then where the } \\
\text { variable, a, has inevitably the relationship with the variable, c. }\end{array}$ \\
\hline 5: Decision of levels & $\begin{array}{l}\text { Transitivity matrix is converted into the canonical matrix format } \\
\text { which arranges elements by levels. }\end{array}$ \\
\hline $\begin{array}{l}\text { 6: Building of ISM } \\
\text { model }\end{array}$ & $\begin{array}{l}\text { Variables in each level identify the connection among risks based on } \\
\text { the relationship defined by SSIM. }\end{array}$ \\
\hline
\end{tabular}

Table 2 which is described as Stage 2 is the algorithm which determines weight values by supply chain experts utilizing relevant information proposed in Stage 1 and applies Grey approach $[20,21,23,26]$.

Table 2. Stage 2. The Algorithm of Grey Approach

\begin{tabular}{|c|l|}
\hline Step & \multicolumn{1}{|c|}{ Explanation } \\
\hline 1 & $\begin{array}{l}\text { Gathering of decision makers' opinion about attributes and } \\
\text { alternatives }\end{array}$ \\
\hline 2 & Evaluation of Grey weight of each attribute \\
\hline 3 & Evaluation of attribute rating value of each alternative \\
\hline 4 & Establishment of Grey weighted average and Grey decision matrix \\
\hline 5 & Normalization of Grey decision matrix \\
\hline 6 & Establishment of the weighted and normalized Grey decision matrix \\
\hline 7 & Making of the ideal alternative \\
\hline 8 & Calculation of the Grey possibility \\
\hline 9 & Ranking the order of the alternatives \\
\hline
\end{tabular}




\section{Experiment}

The risks which destroy a supply chain are various. This paper proposes a method to find the most vulnerable position for 8 risks represent the most frequent in the investigation of Business Continuity Institute Survey [15]. The selected 8 risks are summarized in the following Table 3. This study aims to extend the study of Park [27] and describe in detail the study.

Table 3. The Selected Risk Attribute

\begin{tabular}{|c|l|}
\hline Risk \# & \multicolumn{1}{|c|}{ Attribute } \\
\hline R1 & Unplanned IT \& telecommunications outage (72\%) \\
\hline R2 & Cyberattack \& data breach (50\%) \\
\hline R3 & Adverse weather (41\%) \\
\hline R4 & Transport network or disruption (38\%) \\
\hline R5 & Loss of talent/skills (36\%) \\
\hline R6 & Outsourcer failure (34\%) \\
\hline R7 & New laws or regulation (31\%) \\
\hline R8 & Product quality incident (e.g. human recall) (29\%) \\
\hline
\end{tabular}

The process which determines the position that the selected 8 risks give a supply chain the fatal effect applies the ISM of Stage 1 and the Grey approach of Stage 2. The explanation is as follows. Supply chain experts evaluate subjectively importance, destructive power, continuity, and etc. However, the risk arising in a supply chain should be evaluated objectively if possible and then the result is agreed by all. Therefore, ISM steps of Stage 1 give experts the relevant information of the selected risks before applying Grey approach and select objective grey numbers about linguistic variables. Finally, the ISM algorithm makes the relationship map shown on Figure 4.

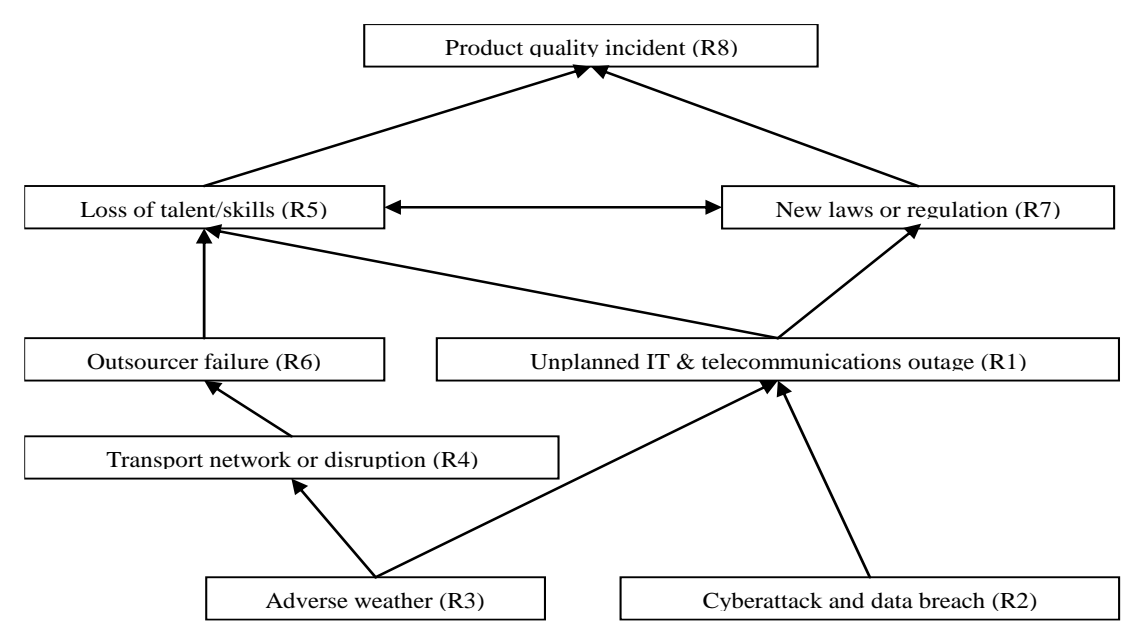

Figure 4. ISM for Risk Relationship in a Supply Chain

This study applies Grey approach considering the risk relationship of Figure 4 obtained by ISM of Stage 2. It is supposed that supply chain risk experts are 4 members and the supply chain consists of 6 tiers (Group $1 \sim$ Group 6). Supply chain reflects Figure 4 and evaluates Grey number into Table 4 and 5 about risk seriousness. 
Table 4. The Linguistic Attribute Weights in Grey Numbers

\begin{tabular}{|c|c|c|}
\hline \multicolumn{3}{|c|}{ The seriousness of attribute } \\
\hline Linguistic variables & \multicolumn{2}{|c|}{ grey numbers } \\
\hline Very Low (VL) & 0.0 & 0.2 \\
\hline Low (L) & 0.2 & 0.4 \\
\hline Medium Low (ML) & 0.4 & 0.5 \\
\hline Medium (M) & 0.5 & 0.6 \\
\hline Medium High (MH) & 0.6 & 0.8 \\
\hline High (H) & 0.8 & 0.9 \\
\hline Very High (VH) & 0.9 & 1.0 \\
\hline
\end{tabular}

Table 5. The Attribute Ratings Value of Alternatives in Grey Numbers

\begin{tabular}{|c|c|c|}
\hline \multicolumn{3}{|c|}{ The rating value of alternative } \\
\hline $\begin{array}{c}\text { Linguistic variables for risk index } \\
\text { (preventable risk } \times \text { resulting risk) }\end{array}$ & \multicolumn{2}{|c|}{ grey numbers } \\
\hline None (N) & 0 & 2 \\
\hline Pretty Rare (PR) & 2 & 4 \\
\hline Rare (R) & 4 & 5 \\
\hline Usual (U) & 5 & 6 \\
\hline Serious (S) & 6 & 8 \\
\hline Very Serious (VS) & 8 & 9 \\
\hline Uncontrollable (UC) & 9 & 10 \\
\hline
\end{tabular}

Table 4 and 5 which are decided by the experts of a supply chain help that this study connects the ambiguity and uncertainty which are the properties of the risks of a supply chain to fuzzy concept and gives the quantitative result. The impact of a risk is not easy quantified in a supply chain because a risk which occurs in a supply chain influences on the different results to the supply chain by location, time, response strategy, response time, and etc. Therefore, this paper applies Grey approach in order to consider fuzzy information which reflects the risk of a supply chain and to make the result.

Also, because the result is varied by the impact of the risks in a supply chain, this study gives information which considers the relationship among risks using ISM and decides effectively Grey numbers.

This paper applies the Grey approach of Table 2 using Table 4 and 5. And then the most vulnerable tier about the proposed risks is T1 among 6 tiers. After that it is vulnerable to risks of $\mathrm{T} 5, \mathrm{~T} 4, \mathrm{~T} 3, \mathrm{~T} 2$, and $\mathrm{T} 6$ order.

\section{Conclusions and Further Studies}

The This study proposed a method to determine the priority of locations (tiers) which should respond first of all for the risks which arise in various forms by various causes in a supply chain. The assessment of the risks arising from a supply chain gives different results by a variety of indicators such as characteristics of supply chain experts, supply chain environment, associations among risks, and etc. Therefore, this study objectified the risks which occur in a supply chain within the possible range and then proposed a method to find the place that have the greatest impact.

This study applied ISM to consider the impact of risks which occur in a supply chain. The method is a process which is defined as visible and well-defined models useful for many purposes about unclear and poorly articulated mental models of systems. And if it is 
used to evaluate risks in a supply chain, it is taken to account the interdependency between the various risks because it is possible to show a driver risk of multiple other risks.

Because the risks arising from a supply chain itself are ambiguous and an evaluator gives subjective result for risks, this paper applied Grey approach to find the weakest position at risks while ensuring the objectivity. The method presented the opinion of supply chain experts about risks in an objective value that Grey numbers using ISM and Grey approach. Using this value, the method finds the location which is the weakest position to next and responds it.

This study presented sequentially one by one from the most vulnerable location to the nest. However, it is necessary to present objectively that the most vulnerable location is how much more vulnerable compared to the next weaker position. So, this paper proposes it to further study.

\section{Acknowledgments}

This study was conducted by research funds from Gwangju University in 2017.

\section{References}

[1] P. R. Erlich and A. H. Ehrlich, "The Population Explosion", Touchstone, New York, (1991).

[2] M. Starik and G. P. Rands, Editor, "Weaving an Integrated Web: Multilevel and Multisystem Perspectives of Ecologically Sustainable Organizations", Academy of Management Review, vol. 20, no. 4, (1995), pp. 908-935.

[3] S. K. Sikdar, "Sustainable Development and Sustainability Metrics", AIChE Journal, vol. 49, no. 8, (2003), pp. 1928-1932.

[4] J. Elkington, "Enter the Triple Bottom Line", Edited A. Henriques and J. Richardson, Earthscan, London, (2004), pp. 1-16.

[5] C. R. Carter and D. S. Rogers, "A Framework of Sustainable Supply Chain Management: Moving Toward New Theory", International Journal of Physical Distribution \& Logistics Management, vol. 38, no. 5 , (2008), pp. 360-387.

[6] I. Heckmann, T. Comes and S. Nickel, "A Critical Review on Supply Chain Risk - Definition, Measure and Modeling", Omega, vol. 52, (2015), pp. 119-132.

[7] J. G. March and Z. Shapira, "Managerial Perspectives on Risk and Risk Taking", Management Science, vol. 33, no. 11, (1987), pp. 1404-1418.

[8] H. Peck, "Reconciling Supply Chain Vulnerability, Risk and Supply Chain Management", International Journal of Logistics Research and Applications, vol. 9, no. 2, (2006), pp. 127-142.

[9] V. G. Venkatesh, S. Rathi and S. Patwa, "Analysis on Supply Chain Risks in Indian Apparel Retail Chains and Proposal of Risk Prioritization Model using Interpretive Structural Modeling", Journal of Retailing and Consumer Services, vol. 26, (2015), pp. 153-167.

[10] F. H. Knight, Editor, "Risk, Uncertainty and Profit", Courier Dover Publications, Mineola, NY, (2012).

[11] J. F. Yates and E. R. Stone, "Risk appraisal", Edited J. F. Yates, John Wiley, Chichester, U.K., (1992).

[12] M. Christopher, H. Peck, C. Rutherford and U. J. Jüttner, "Understanding Supply Chain Risk: a SelfAssessment Workbook", Department for Transport - Cranfield University (2003).

[13] F. Wiengarten, P. Humphreys, C. Gimenez and R. McIvor, "Risk, Risk Management Practices, and the Success of Supply Chain Integration", International Journal of Production Economics, vol. 171, no. 3, (2016), pp. 361-370.

[14] R. Gaonkar and N. Viswanadham, "A Conceptual and Analytical Framework for the Management of Risk in Supply Chains", Proceedings of the 2004 IEEE international Conference on Robotics \& Automation, New Orleans, LA, (2004) April 26- 1 May.

[15] Business Continuity Institute, "Supply Chain Resilience Report 2015", http://www.bcifiles.com/bcisupply-chain-resilience-2015.pdf (2015).

[16] O. Khan, M. Christopher and B. Burnes, "The Impact of Product Design on Supply Chain Risk: A Case Study", International Journal of Physical Distribution \& Logistics Management, vol. 38, no. 5, (2008), pp. 412-432.

[17] Sushil, "Interpreting the Interpretive Structural Model", Global Journal of Flexible Systems Management, vol. 13, no. 2, (2012), pp. 87-106.

[18] G. G. Lendaris, "Structural Modelling- A Tutorial Guide", IEEE Transactions on Systems, Man, and Cybernetics, vol. 10, no. 2, (1980), pp. 807-840. 
[19] W. Hachicha and M. Elmsalmi, "An Integrated Approach Based-Structural Modeling for Risk Prioritization in Supply Network Management", Journal of Risk Research, vol. 17, no. 10, (2014), pp. 124.

[20] D. Jelong, "Introduction to Grey System Theory", The Journal of Grey System, vol. 1, (1989), pp. 1-24.

[21] D. Jelong, "Grey System Theory", Huazhong University of Science and Technology Press, Wuhan, China, (2002).

[22] M. S. Memon, Y. H. Lee and S. I. Mari, "Group Multi-Attribute Supplier Selection using Combined Grey Systems Theory and Uncertainty Theory", Expert Systems with Applications, vol. 42, (2015), pp. 7951-7959.

[23] V. Baskaran, S. Nachiappan and S. Rahman, "Indian Textile Suppliers' Sustainability Evaluation using the Grey Approach", International Journal of Production Economics, vol. 135, no. 2, (2012), pp. 647-658

[24] A. J. Brito, A. T. Almeida and C. M. M. Mota, "A Multi Attribute Model for Risk Sorting of Natural Gas Pipelines Based on ELECTRE TRI Integrating Utility Theory", European Journal of Operational Research, vol. 200, no. 3, (2010), pp. 812-821.

[25] S. Jharkharia and R. Shankar, "IT Enablement of Supply Chains: Modeling the Enablers", International Journal of Productivity and Performance Management, vol. 53, no. 8, (2004), pp. 700-712.

[26] G. Li, D. Yamaguchi and M. Nagai, "A Grey-Based Decision-Making Approach to the Supplier Selection Problem", Mathematical and Computer Modelling, vol. 46, no. 3-4, (2007), pp. 573-581.

[27] K. Park, "Decision of Risk Priority of a Supply Chain using ISM and Grey Approach", Asia-pacific Proceedings of Applied Science and Engineering for Better Human Life, (2016); Jeju, Korea.

\section{Author}

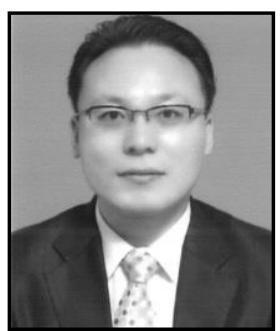

KyoungJong Park, $\mathrm{He}$ is a Professor in the Department of Business Administration, Gwangju University, Korea. He received his B.Sc., M.Sc., and Ph.D. from the Department of Industrial Engineering, Hanyang University, Korea in 1992, 1994, and 1998. He spent a sabbatical year at Pennsylvania State University, 20102011. His research interests are Fuzzy Information, Evolutionary Algorithm, Simulation Optimization, and Service Supply Chain Management. 
International Journal of $u-$ and $\mathrm{e}-$ Service, Science and Technology Vol.10, No.9 (2017) 